\title{
Persistent ectopic pregnancy following ipsilateral "salpingectomy"
}

\author{
M. I. Rizzuto $\cdot$ R. Macrae $\cdot$ F. Odejinmi
}

Received: 27 January 2007 / Accepted: 10 May 2007 / Published online: 12 June 2007

(C) Springer-Verlag 2007

\begin{abstract}
Persistent ectopic pregnancy (PEP) following ipsilateral "salpingectomy" is a rare occurrence. This report describes this uncommon condition in a 26-year-old woman who presented with a sudden onset of right fossa iliac pain following an earlier salpingectomy. At laparoscopy, a persistent ipsilateral EP in the right fallopian tube stump was found. At the initial laparoscopy, an endoloop was used for salpingectomy, and a tubal stump of about $4 \mathrm{~cm}$ was left. This poses the questions: Are salpingectomies performed with endoloops true salpingectomies or partial salpingectomies? Do women managed with endoloops need to have human chorionic gonadotropin (hCG) follow-up, as do those with salpingostomy?
\end{abstract}

Keywords Ectopic pregnancy - Laparoscopy ·

Salpingectomy

\section{Introduction}

Ectopic pregnancy (EP) constitutes one of the major gynaecological emergencies. In the UK, there are around 11,000 cases of EP per year (incidence 11.5 per 1,000 pregnancies), with 11 deaths in the last triennium [1]. Persistent ectopic pregnancy (PEP) can present as a more serious health risk than classical EP, with up to $24 \%$ rupturing in the postoperative period after conservative

M. I. Rizzuto $(\square) \cdot$ R. Macrae $\cdot$ F. Odejinmi $(\square)$

Department of Obstetrics and Gynaecology,

Whipps Cross University Hospital,

Whipps Cross RD, Leytonstone,

London E11 1NR, UK

e-mail: ivanarizzuto@tiscali.it

e-mail: jimi@doctors.org.uk therapy of EP [2]. The majority of EP patients present subacutely, and diagnosis is usually made using algorithms that include a combination of symptoms, ultrasound scans and beta human chorionic gonadotropin ( $\beta-\mathrm{hCG})$. Though it is possible to manage certain cases conservatively or medically, for the majority of women, surgery remains the mainstay of treatment, which may be radical (salpingectomy) or conservative (salpingostomy), with operative laparoscopy being the gold standard. It is advised that if the contralateral tube is damaged salpingostomy be performed, but this may be associated with persistent trophoblast, and women need to be followed up with serial hCG estimations. Salpingectomies on the other hand are performed when the contralateral tube is normal, using either manufactured pretied knots (endoloops) or diathermy. Because the tube is removed, there should be no need for serial hCG estimations.

We present a rare case of a PEP, occurring in the proximal remnant of the right fallopian tube, after an ipsilateral "salpingectomy". The aim of our report highlights the possible drawbacks of using endoloops for salpingectomy, as a longer tubal stump remains and may be the source of either persistent or recurrent EP.

\section{Case report}

A 26-year-old woman with a history of two pregnancy terminations and one normal delivery presented to our gynaecological emergency service complaining of right iliac fossa pain of acute onset, which was colicky, radiating to the shoulder and associated with vaginal bleeding. Though she was unsure of her dates, her urinary pregnancy test was positive, and ultrasound scan showed a right-sided heterogeneous adnexal mass (size $23 \times 33 \mathrm{~mm}$ ) with free 


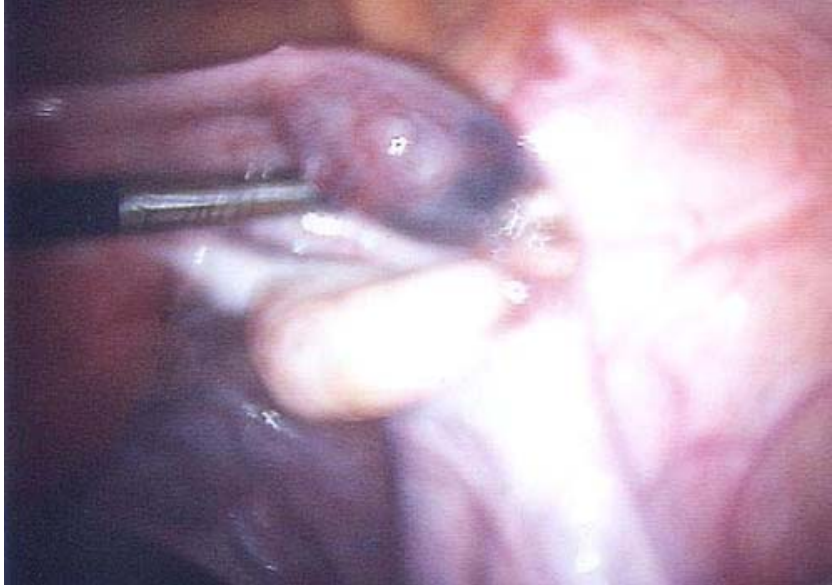

Fig. 1 Persistent ectopic pregnancy in the stump left from previous salpingectomy

fluid in the pouch of Douglas. Her serum hCG, however, was only $436 \mathrm{IU} / 1$.

Because of the severity of her symptoms, she underwent an emergency laparoscopy at which the scan findings were confirmed. She thus underwent a right salpingectomy performed with two endoloops. The procedure was uneventful, and she was discharged the next day. Twenty-one days, later the patient re-presented with severe pain in the right iliac fossa. Her vital signs were stable, and her urine pregnancy test was positive. Her $\beta-h C G$ was 8925 IU/l. Transvaginal ultrasound scan revealed an empty uterus and a right heterogeneous adnexal mass. Despite her "salpingectomy" she underwent repeat laparoscopy, and a PEP was found in the proximal tubal stump (Fig. 1). This was removed using bipolar diathermy and scissors.

Histological report of the specimen showed trophoblastic tissue within the fallopian tube lumen, thus confirming the tubal ectopic pregnancy. The patient recovered from the surgery uneventfully and was discharged on day 2 postoperation.

\section{Discussion}

Laparoscopy has become the gold standard for the surgical management of ectopic pregnancy. The problem with salpingostomy is persistent trophoblastic tissue, the incidence of which has been reported to be between $3 \%$ and $20 \%$ [3]. It is recommended that women should be followed up with serial hCG to prevent morbidity from this complication. There are no such reports following salpingectomy and thus no such recommendations. There are reports in literature of recurrent EP in the ipsilateral fallopian tube following salpingectomy or partial salpingectomy $[4,5]$ but none of PEP. This may be because such negative complications are not publicised, but herein we believe lies a valuable lesson. In our case, at the first presentation, a right "salpingectomy" was performed using endoloops. However, this resulted in a PEP in the tubal stump.

Salpingectomy can either be carried out using electrosurgical techniques or by use of endoloops. The dangers of electrosurgery cannot be overemphasised, with burns as a complication being the second largest notification to the medical defence union second only to laparoscopic entry complications [6]. Modern operative laparoscopy strives to eliminate this risk, and it is for this reason that the endoloops may be superior to electrosurgery, particularly in residency programmes. Endoloops have been shown to eliminate this risk and do not significantly add to the length of surgery when used for sterilisation in a residency programme. It also allows junior laparoscopists to develop operative laparoscopic skills and gain experience in methods commonly used during more difficult cases in a controlled and manageable environment [7].

On the other hand, removal of the whole tube with an endoloop can sometimes be technically difficult because of adhesions or the proximity of the ovary to the tube. Therefore, it is suggested that diathermy and laparoscopic scissors be used to excise the proximal tube, followed by an endoloop to remove the EP to reduce the risk of persistent or recurrent EP at the tubal stump [8]. This approach, however, still exposes the patient to the risk of burns. An alternative approach could be the use of one endoloop to remove the ectopic and another to remove the tubal stump during the same operation, thus eliminating both risks, i.e. that of electrosurgery and of persistent trophoblast. Another approach would be to follow up with serial hCG all women who have had salpingectomy. In our opinion, this would not be cost effective, as this is the first complication of this kind in more than 300 EPs managed by salpingectomy over 5 years in our institution (personal communication).

In conclusion, partial laparoscopic salpingectomies should be avoided, as total salpingectomies can be undertaken safely with minimal risk to the patient. Our case also illustrates the importance of maintaining a high index of suspicion for the diagnosis of PEP, even after salpingectomy, especially when endoloops are used.

\section{References}

1. Why women die. Confidential enquires into maternal deaths in the United Kingdom 2000-2002

2. Graczykowski JW, Mishell DR Jr (1997) Methotrexate prophylaxis of persistent ectopic pregnancy after conservative treatment by salpingostomy. Obstet Gynecol 89:118-122

3. Yao M, Tulandi T (1997) Current status of surgical and nonsurgical management of ectopic pregnancy. Fert Steril 67(3):421-433 
4. Murray H, Baakdah H, Bardell T, Togas T (2005) Diagnosis and treatment of ectopic pregnancy. CMAJ 173(8):905-912

5. Zuzarte R, Khong CC (2005) Recurrent ectopic pregnancy following ipsilateral partial salpingectomy. Singapore Med J 46(9):476-478

6. Goodwin H (1998) Minimal access surgery. Journal of the MDU 14(1):13-14
7. Parson MT, Hill DA (1994) The benefits of using the loop ligature (endoloop) Laparoscopic sterilization procedure in a residency program. J Gynecol Surg (10):15-19

8. Sherif LS, Foda AI, El Zayat MM, Badawy AM, Magos AL (1999) Laparoscopic salpingectomy:electrosurgery or suture? Gynaecol Endosc (8):80-93 